\title{
Lettre ouverte à Monsieur Didier Burkhalter, Conseiller Fédéral
}

Groupe de presse de l'ANMO

Correspondance:

Dr Joël Rilliot

Groupe de presse de l'ANMO

Rue de la Fin 14

CH-2016 Cortaillod

\section{Monsieur le Conseiller Fédéral,}

Permettez-nous tout d'abord, au nom de l'Association Neuchâteloise des Médecins Omnipraticiens (ANMO) de vous féliciter pour votre élection. C'est en tant que groupe de réflexion issu de l'ANMO que nous nous adressons à vous par le biais d'une lettre ouverte. Au moment de votre entrée en fonction, nous tenons à vous faire part de notre vécu de médecin de famille, ceci sans arrière-pensée corporatiste ni défense d'un quelconque privilège.

Nous avons le sentiment que les milieux politiques et économiques ont une perception fragmentaire et erronée de l'activité du médecin généraliste. Ils affirment pourtant qu'il est la pierre angulaire d'un système de santé équilibré. Mais quelle image ont-ils de ce médecin? Ils le réduisent presque exclusivement à une fonction de trieur ou de gestionnaire des urgences. Rôle qui pourrait, selon eux, se satisfaire d'une formation accélérée ou, à la rigueur, être tenu par des non médecins, voire par une permanence téléphonique ... C'est oublier que le tri et l'urgence, pour être efficaces et économiquement pertinents, doivent être effectués par des professionnels d'expérience. Mais c'est surtout
Qui pourra raisonnablement quantifier ce que coûte une angine, la découverte d'un diabète, la perte de son travail ou le conflit avec son conjoint? L'activité du médecin généraliste n'est donc pas, à l'évidence, appréciable selon les critères économiques habituels.

Avoir un médecin de famille répond à une attente prioritaire de la population. Développer avec lui une relation de confiance, s'inscrivant dans la durée, ne pourra jamais être remplacé par un conseil téléphonique ou par une approche technique. De nombreuses études, dans divers pays, ont démontré que le meilleur moyen d'assurer les soins au meilleur coût nécessite un réseau important de médecins de famille (environ 1 généraliste pour 1 spécialiste). En Suisse, nous n'en sommes qu'à environ 1 généraliste pour 3 spécialistes. Et les attaques multiples ainsi que la dépréciation continue dont la profession est régulièrement victime ces dernières années ne contribuent pas à la rendre attractive: moratoire sur l'ouverture de cabinets, frein du laboratoire du praticien, tarification TARMED qui creuse le fossé entre généralistes et spécialistes, attitude de plus en plus soupçonneuse des assureurs vis-à-vis de leurs partenaires médecins et le peu de cas qui est

\section{Nous avons le sentiment que les milieux politiques et économiques ont une perception fragmentaire et erronée de l'activité du médecin généraliste}

ignorer que tri et urgences ne représentent que 15\% de l'activité du généraliste. En effet, pour l'essentiel, il gère des patients dans leur globalité. C'est lui qui assume les maladies chroniques, devenues dans notre société le principal motif de consultation. C'est à lui de prendre en charge les problèmes existentiels, de plus en plus fréquents aujourd'hui, qu'ils soient familiaux ou socioprofessionnels C'est à lui que revient la tâche d'expliquer le but puis de discuter les résultats des examens sophistiqués qu'offre la médecine actuelle. C'est lui encore qui assume le suivi de ses patients après une hospitalisation. C'est à lui qu'incombe la tâche d'annoncer les diagnostics sévères, puis d'assurer l'accompagnement, souvent jusqu'à la mort. Chaque patient est différent, chaque maladie est vécue différemment.

Comment dès lors parler de concurrence, d'évaluation économique dans une activité aussi complexe? fait de leur avis. S'y ajoute l'attrait de la société pour les prouesses techniques abondamment véhiculées par les médias. Pourtant elles ne concernent qu'une minorité de patients puisqu'une seule personne sur mille consultant un généraliste aboutira dans un hôpital universitaire.

Une réelle promotion de la médecine de terrain passe par l'augmentation de la relève. Il faut privilégier cette formation tant à l'université que dans les cabinets privés. Les médecins généralistes romands concrétisent ces propositions par la création du cursus romand de médecine générale (www.devenirgeneraliste.ch). C'est aussi un des objectifs majeur de l'initiative populaire que la Société suisse de Médecine générale vient de lancer.

Les soins en réseau (Managed Care), promus au rang de solutions à tous les maux, ne peuvent s'appli- 
quer que si le nombre de médecins omnipraticiens est élevé. Dans le cas contraire, cette proposition est vide de sens.

Enfin il faut évoquer le rôle délétère des caisses maladie, qui voient le plus souvent les médecins comme des adversaires, voire des profiteurs, cherchant à tirer parti de leur rôle social. Elles visent à prendre le pouvoir de la gestion des soins: elles souhaitent la suppression de l'obligation de contracter, planifient l'organisation de réseaux dont elles choisiraient ellesmêmes les médecins, multiplient les demandes de renseignements médicaux, souvent inutiles et arbitraires. Le temps des mutuelles est bien passé. Elles sont devenues des entreprises commerciales. Leur concurrence est factice et onéreuse, sauf dans la recherche des «bons cas», jeunes, en bonne santé, présentant peu de risques. Et que dire de l'opacité de leur comptabilité? Alors que selon la LaMal elles n'ont pas le droit de faire de profit! Quels sont les flux d'argent entre l'assurance de base et les complémentaires? Que penser de leurs réserves? Qui sont calculées sur l'ensemble de leurs assurés en Suisse, alors que les primes sont fixées par cantons ou par région. Et n'est-il pas absurde que lors d'un changement, les réserves d'un assuré restent acquises à la caisse qu'il quitte? Perdre des clients permet d'améliorer le bilan des réserves! Et comment l'OFSP peut-il contrôler valablement les comptes et les primes de chaque caisse, dans chaque canton?
Nous adhérons à la proposition de la Société médicale de la Suisse romande de séparer clairement l'assurance de base des assurances complémentaires en interdisant à un assureur de pratiquer ces deux types d'activité. Déjà remise à Pascal Couchepin en 2003 dans le cadre d'un manifeste intitulé «Neuf pistes pour améliorer l'économicité et la qualité du système de suisse de santé», cette proposition n'avait obtenu aucun écho. Nous sommes heureux de voir qu'elle se met à susciter récemment des discussions, dans les milieux politiques et économiques. Elle est, à l'évidence, la seule à pouvoir amener de la clarté dans l'application de la LaMal. C'est l'occasion de rappeler aussi les autres pistes évoquées par ce manifeste, qui témoignent de la volonté des médecins de premier recours de mettre leurs forces au service de la maîtrise des coûts de santé.

Nous vous remercions de l'attention que vous accorderez à nos propos. Nous sommes ouverts à toute remarque ou objection et demeurons à votre disposition pour d'éventuels compléments d'information ou pour vous rencontrer.

Nous vous adressons, Monsieur le Conseiller Fédéral, nos respectueuses salutations.

Groupe de Presse de I'ANMO

Drs Jean-François Boudry, Gilbert Villard, Joël Rilliot et Jean-Paul Studer

\section{Sie lesen gerade eine Zeitschrift des Schweizerischen Ärzteverlags EMH}

\section{Wussten Sie schon,}

- dass EMH ein Gemeinschaftsunternehmen der Verbindung der Schweizer Ärztinnen und Ärzte FMH und der Schwabe AG ist, dem mit Gründung 1488 ältesten Druck- und Verlagshaus der Welt?

dass EMH mit insgesamt zehn Fachzeitschriften, einem umfangreichen Online-Angebot sowie einem wachsenden Buchprogramm der führende Verlag für medizinische Zeitschriften in der Schweiz ist?

- dass sämtliche bei EMH erscheinenden Zeitschriften offizielle Publikationsorgane der jeweils zuständigen medizinischen Fachorganisationen sind?

Wenn Sie mehr über EMH wissen möchten, finden Sie unter www.emh.ch weitere Informationen.

EMH Schweizerischer Ärzteverlag - Publikationen am Puls der Medizin 